provide controlled and reversible effects, but also either inhibition or enhancement of movement. Data are accumulating in support of a biphasic influence of cell/ substratum adhesiveness on migration speed $^{10}$. So, depending on the level of cell-surface integrin and corresponding extracellular-matrix ligand, interactions between proteinase/inhibitor pairs such as UPA/PAI-1 could alter the cell/substratum adhesiveness, either towards or away from that yielding maximal migration. Experimental evidence consistent with this bidirectional control has been found in a simpler model system involving a soluble integrin-binding competitor ${ }^{11}$.

Both of these papers ${ }^{1,2}$ illustrate the central importance of the physical processes underlying cell locomotion ${ }^{12,13}$, and show how molecular interactions can influence the linkages between the matrix, the receptor and the cytoskeletal motor. Ground for future explorations will thus be found in understanding how these linkages are regulated biochemically, for instance by growthfactor signalling, and especially how this regulation might provide physical asymmetries in force transmission across the length of the cell to permit forward movement of the cell body ${ }^{5,12}$.

Douglas A. Lauffenburger is in the Department of Chemical Engineering and Center for Biomedical Engineering, Massachusetts institute of Technology, Cambridge, Massachusetts 02139, USA.

\title{
Revolution in magneto-optics
}

\section{J. A. C. Bland}

WHEN light is reflected from the surface of a ferromagnet, its plane of polarization rotates. This magneto-optical Kerr rotation has been with us for a long time, having first been discovered in the late nineteenth century ${ }^{1}$, but so far the size of the rotation we can produce remains disappointingly small - typically a few degrees, which is much the same as with the polished magnet pole-pieces of Kerr's original experiment. As a result, only a small optical signal can be generated by reversing the material's magnetization with an external field. (Even so, the advent of the laser and advanced signal detection methods has made it possible to detect even tiny Kerr rotations, and the effect is already used in rare-earth/transition-metal alloy films for erasable information storage.) But now a remarkable 'giant' Kerr rotation through $90^{\circ}$ has been observed by Pittini et al. ${ }^{2}$ in a single cerium-antimony crystal. The peak effect occurs for incident infrared light with an energy of 0.46 electron volts.

Not only is this the first time that such a large Kerr effect has been seen (the previous record being $14^{\circ}$, in the same material ${ }^{3}$ ), but no theoretical prediction of such a large effect has been made, despite several investigations of ceriumand antimony-based compounds ${ }^{4}$. By definition, the largest Kerr rotation possible is $90^{\circ}$, as it is the oscillating electric field vector that is rotated and so $+90^{\circ}$ and $-90^{\circ}$ are equivalent. This rotation is so large that it renders invalid the usual theoretical approximations, which relate the polarization change to the off-diagonal terms in a material's conductivity matrix (those that represent currents induced perpendicular to an applied electric field).

From the point of view of applications, big is good, so of course giant would be better. Much research effort has been concen- trated on developing magnetic multilayer materials, such as those based on ultrathin cobalt-platinum layers (a few ångströms thick) which show sizeable Kerr rotations at the ultraviolet wavelengths needed for high-density recording applications. (A laser reads the rotation produced by each

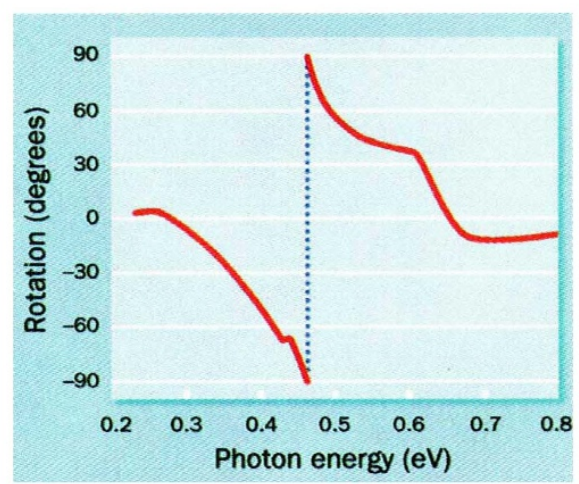

The giant rotation of polarized light on reflection from a cerium-antimony crystal (from ref. 2).

magnetic domain, and so the resolution is determined by the laser wavelength.)

Other magnetic properties are required for magneto-optical recording applications: in 'perpendicular recording', there must be an internal magnetic field (the 'anisotropy field') favouring a magnetization perpendicular to the magnetic film plane; and in thermomagnetic recording, the Curie temperature (above which the magnetization is lost) must be tuneable, so as to allow 'writing' by a laser pulse, and the stability of the magnetization to external fields must be large enough at room temperature to prevent the medium from being demagnetized.

The finding of Pittini and co-workers is unlikely to revolutionize magneto-optical recording, however, requiring as it does a single crystal, high fields (five tesla) and low temperatures $(1.5 \mathrm{~K})$. Moreover, the $0.46-\mathrm{eV}$ energy of the peak magneto-optical rotation requires a reading wavelength of more than a micrometre - too long to be of use in high-density recording. In general, the size of the rotation is reduced in polycrystalline materials compared with single crystals, so thin films of $\mathrm{CeSb}$ will certainly show a smaller effect. Nevertheless, it is conceivable that a niche application could be found for $\mathrm{CeSb}$, such as a field-controllable optical isolator or switch, which requires such a large optical rotation.

There was already a hint that a large rotation existed in CeSb: in 1986, Reim et $a l^{3}$ saw that the Kerr effect increased as the photon energy was reduced. It still rose as the energy was reduced to $0.5 \mathrm{eV}$, and showed no sign of a maximum, but with their apparatus they couldn't reach the all-important energy region.

In part, the giant effect in $\mathrm{CeSb}$ can be explained as a fortunate concurrence of several effects. At $0.46 \mathrm{eV}$ there is a 'plasma minimum': the incoming radiation excites a collective motion of the electrons, which screens the electrical polarization inside the material. That reduces those components of the reflectivity that do not change the polarization of incident radiation, so a large rotation is usually seen near a plasma minimum ${ }^{5}$. Microscopically, the Kerr effect arises from spin-dependent transitions between initial and final electron states. The lower level involved in the magneto-optical transition in $\mathrm{CeSb}$ is a 'saturated state', that is, its component of angular momentum parallel to an external field has the most negative value possible, and so the state can only absorb righthanded circularly polarized light (which increases the angular momentum).

But these factors can only explain part of the rotation. If we are to explain the giant effect fully, we must understand the details of the transition much better than we do: in particular, the precise nature of the $4 f$ state in CeSb needs to be described better. The key issue is how it differs from the same state in the isolated cerium atom, owing to electronic interactions.

The $90^{\circ}$ rotation found by Pittini et al. challenges our understanding of these rare-earth compounds, and new theoretical efforts are needed to describe the magneto-optics of $\mathrm{CeSb}$ and related materials. Such an effort may in turn play an important part in the search for other magneto-optical materials suitable for future applications.

\section{J. A. C. Bland is at the Cavendish Labora- tory, University of Cambridge, Cambridge CB3 OHE, UK.}

\footnotetext{
1. Kerr, J. Phil. Mag. 3, 321 (1877).

2. Pittini, R. et al. Phys. Rev. Lett. 77, 944 (1996).

3. Reim, W. et al. J. Magn, Magn. Mater. 54-57, 1401 (1986).

4. Antropov, V. P. et al. J. Magn. Magn. Mater. 140-144, 1161 (1995).

5. Takanashi, K. et al. Jap. J. Appt. Phys. 29, L306 (1990).
} 